\title{
The Baboon as a Good Model for Studies of Human Kidney Development
}

\author{
LINA GUBHAJU AND M. JANE BLACK \\ Department of Anatomy and Cell Biology, Monash University, Clayton, Victoria 3800, Australia
}

\begin{abstract}
ABST
Because of the improved survival of premature neonates in
recent years, it is important to investigate the effects of premature
delivery on the kidney, in which nephrogenesis is still ongoing
during the third trimester. Hence, an appropriate animal model
that is similar to humans is essential. The aim of the current study
is to determine the time course of nephrogenesis in the baboon,
to establish whether it is a suitable model of human nephrogen-
esis. At the Southwest Foundation for Biomedical Research (San
Antonio, TX), fetal baboons were delivered prematurely by
cesarean delivery and at term by natural delivery. Fixed kidneys
from 125-, 140-, 175-, and 185-d gestation baboons were as-
sessed morphologically for evidence of a nephrogenic zone.
Nephron number, kidney volume, and glomerular and corpuscle
volume were also estimated using unbiased stereology. Morpho-
\end{abstract}
logic assessment confirmed the presence of metanephric mesenchyme and immature glomeruli in the nephrogenic zone of the kidneys from the prematurely delivered fetuses at 125 and $140 \mathrm{~d}$ gestation. At $175 \mathrm{~d}$ gestation and at term, the nephrons seemed to be mature. Both kidney weight $\left(R^{2}=0.918, p=0.0002\right)$ and kidney volume $\left(R^{2}=0.837, p=0.001\right)$ were very strongly correlated with nephron number. There was also a direct relationship between gestational age $\left(R^{2}=0.589, p=0.03\right)$ and birth weight $\left(R^{2}=0.562, p=0.03\right)$ with nephron number. In conclusion, in this study, nephrogenesis in the baboon is complete before term by $175 \mathrm{~d}$ gestation, which is similar to humans. Hence, the baboon is a suitable model for future studies to investigate human kidney development. (Pediatr Res 58: 505509, 2005)

The incidence of premature delivery has increased substantially in the past decade, such that $6-10 \%$ of all births in the Western world are premature (1). Moreover, as a result of marked advances in neonatal care, infants who are born as early as 26 wk gestation currently have an $80 \%$ chance of survival (2). Therefore, it is imperative to understand how organ development is affected in prematurely delivered individuals, especially in organs in which the ontogeny normally occurs before birth. For instance, infant respiratory distress syndrome and bronchopulmonary dysplasia are commonly observed in preterm neonates as a result of the immaturity of the lungs as pulmonary development is ongoing until term in the human (3). However, in recent years, neonatologists have been able to overcome respiratory complications to some extent by enhancing postnatal lung development, thus increasing survival of premature infants.

Renal development is also rapidly occurring during the third trimester of pregnancy, and so the kidney may also be "at risk" after premature delivery. Indeed, renal failure affects $\sim 8-24 \%$ of all preterm neonates as a result of the immature kidneys (4).

Received October 1, 2004; accepted January 19, 2005.

Correspondence: Lina Gubhaju, Department of Anatomy \& Cell Biology, P.O. Box 13C Monash University, Victoria 3800, Australia; e-mail: Lina.Gubhaju@med.monash.edu.au.

This work was supported by National Institutes of Health grant HL52636, BPD Resource Center grant, and P51RR13986 for facility support.

DOI: 10.1203/01.PDR.0000179397.20862.73
Nephrogenesis, the formation of nephrons, commences at approximately the ninth week of gestation and continues until $\sim 36 \mathrm{wk}$ in humans $(5,6)$; hence, it is probable that nephrogenesis is affected in infants who are born before $36 \mathrm{wk}$ gestation. Nephrogenesis commences with the ureteric bud invading the metanephric mesenchyme, occurring at approximately the fifth week of gestation (5,7-11). Mature nephrons are observed from the ninth week of gestation with $\sim 60 \%$ of all nephrons formed during the third trimester, with no new nephrons formed after birth in term infants $(6,12)$. Therefore, delivery of an infant before term is expected to have an adverse effect on renal development, in particular nephrogenesis.

Given the increasing survival of premature infants, further research is required to investigate the consequences in the kidney. Although rodent models can be studied in larger numbers and are relatively inexpensive, their kidney development is different from humans, with nephrogenesis continuing after birth. Thus, other animal models, including fetal sheep and baboons, have also been very valuable. To date, most of these studies have focused on exploring the effects on the pulmonary and neural systems (13-15). The baboon (Papio species) has served as a very practical model to study the effects of preterm delivery on pulmonary, neural, and renal function, given their close relation to humans and the similarities in the ontogeny of their organs $(14,16-21)$. Use of preterm baboons has enabled researchers to observe the outcomes of 
new therapies such as antenatal corticosteroid treatment in preterm neonates. Although new therapies have improved survival of premature infants by enhancing early organ maturation, it is important to consider the potential detrimental affects on organs as a result of interrupted development in utero.

Although kidney development in humans and some nonhuman primates has been studied $(22,23)$, the time course of nephrogenesis in the baboon remains unknown. For verifying the potential usefulness of the baboon as a model to study the effects of preterm delivery on kidney development, it is essential to determine the time course of nephrogenesis in the baboon and in particular whether nephrogenesis is complete at term. Hence, the major aim of the current study was to explore nephrogenesis in the baboon during normal pregnancy and thus determine whether it is a suitable model to investigate nephrogenesis in humans. In addition, nephron number, kidney volume, glomerular volume, and renal corpuscle volume (comprising the glomerular tuft, Bowman's space, and Bowman's capsule) were estimated using unbiased stereology to determine any potential associations between nephron number and variables such as gestational age, birth weight, and kidney size.

\section{METHODS}

Animals and organ collection. All animal handling and deliveries were performed in the Primate Research Facility at the Southwest Foundation for Biomedical Research (San Antonio, TX). The animal husbandry and animal handling procedures were reviewed and approved to conform to the American Association for Accreditation of Laboratory Animal Care guidelines. Premature fetuses were delivered by cesarean delivery and term animals by natural delivery. Birth weights of all the neonates were obtained after delivery. Animals were killed with sodium pentobarbitone at birth, and the kidneys were collected at necropsy. The kidneys were cleaned of fat and connective tissue, weighed, cut into quarters, and immersion-fixed in $10 \%$ buffered formalin. Kidneys from animals that were delivered at $125(n=2), 140(n=1), 175(n$ $=3$ ), and $185(n=2)$ d gestation (term $\sim 185 \mathrm{~d})$ were used for this study.

Tissue processing, sectioning, and staining. The fixed kidney quarters were sliced into 2-mm slices using a razor blade slicing device. Every second slice of each kidney was processed (dehydrated through graded alcohols) and embedded in glycolmethacrylate resin (Technovit 7100; Kulzers $\mathrm{GmbH}$, Wehrhem, Germany). The number of slices embedded for all kidneys ranged from eight to 11 slices. The glycolmethacrylate blocks then were sectioned exhaustively using a Leica RM 2165 Supercut microtome (Leica Corp., Nusstoch, Germany) at $20 \mu \mathrm{m}$, with every 10th and 11th sections collected (with the first pair randomly chosen). From the remaining kidney slices, three full slices from each kidney that contained a complete cortex and medulla were also processed and embedded in paraffin and cut into 5- $\mu \mathrm{m}$ sections. All collected sections were stained with hematoxylin and eosin.

Morphologic assessment of nephrogenesis. Paraffin sections from all animals were examined for any evidence of ongoing nephrogenesis. The presence of undifferentiated metanephric mesenchyme, the ureteric bud, and developing glomerular structures in the form of comma-shaped and S-shaped bodies in the outer cortex in the nephrogenic zone indicated that glomeruli were still forming and maturing. The presence of only mature glomeruli, with a well-defined glomerular tuft with capillaries and Bowman's capsule, indicated that nephrogenesis was complete.

Estimation of kidney volume, glomerular volume, and glomerular (nephron) number. All 10th glycolmethacrylate sections and the average section thickness were used to calculate kidney volume using the Cavalieri principle (24). Complete 10th and 11th sections (one pair) that were collected and stained were used to estimate nephron number. Complete sections were defined as sections that showed the entire kidney slice without any missing pieces. Using an unbiased physical dissector/fractionator technique, the number of glomeruli (and thereby nephrons) in the kidneys was estimated. Using this technique, the number of nephrons in a known fraction of the kidney was counted to estimate the total number of glomeruli in the whole kidney $(25,26)$. In the counting of nephrons, if immature glomeruli such as S-shaped glomeruli were observed in the sections, then they were excluded so that only mature glomeruli with a well-defined glomerular tuft with open capillaries were counted. These criteria have been previously used to distinguish an S-shaped body from a vascularized glomerulus (10). Glomerular volume and renal corpuscle volume (comprising the glomerular tuft, Bowman's space, and Bowman's capsule) were also determined using well-established, unbiased, stereologic techniques (26).

Statistical analysis. Statistical analyses were performed using GraphPad Prism Version 3.0 for Windows (GraphPad Software, San Diego, CA). Linear regression analyses were performed to determine whether there were significant correlations between nephron number and the following variables: gestational age, birth weight, kidney weight, and kidney volume. The level of significance accepted was $p<0.05$.

\section{RESULTS}

Morphologic assessment of nephrogenesis. Morphologic assessment of premature baboon kidneys at $125 \mathrm{~d}$ gestation showed the presence of developing glomerular structures in the nephrogenic zone located in the superficial outer cortex (Fig. 1). The presence of the wide nephrogenic zone demonstrated that several more generations of nephrons were yet to be formed. More mature glomeruli were observed in the inner regions of the renal cortex. The ureteric bud could also be observed branching into the undifferentiated metanephric mesenchyme. At $140 \mathrm{~d}$ gestation, there was still evidence of new nephrons forming as the ureteric bud and mesenchymal cells were visible in the nephrogenic zone (Fig. 2). Again, it was evident that several more layers of glomeruli were going to form.

By $175 \mathrm{~d}$ gestation, nephrogenesis seemed to be complete, with no evidence of a nephrogenic zone. In kidney sections at $175 \mathrm{~d}$ gestation (Fig. 3), there was no evidence of developing glomerular structures in the renal cortex. The glomeruli also seemed to be mature, with the exception of a few small glomeruli still maturing. Immature glomeruli were smaller in size and seemed to be mainly aggregates of podocytes with not well-developed glomerular capillaries. There was also some indication of maturing tubular structures (refer to Fig. 3). In accordance with our findings at $175 \mathrm{~d}$ gestation, kidney sections from term baboons ( $185 \mathrm{~d}$ gestation) seemed mature (Fig. 4). All glomeruli were large and contained a well-defined glomerular tuft with open capillaries.

Nephron number, kidney volume, and glomerular volume. Using unbiased stereology, nephron number, kidney volume, and glomerular and renal corpuscle volume were estimated.
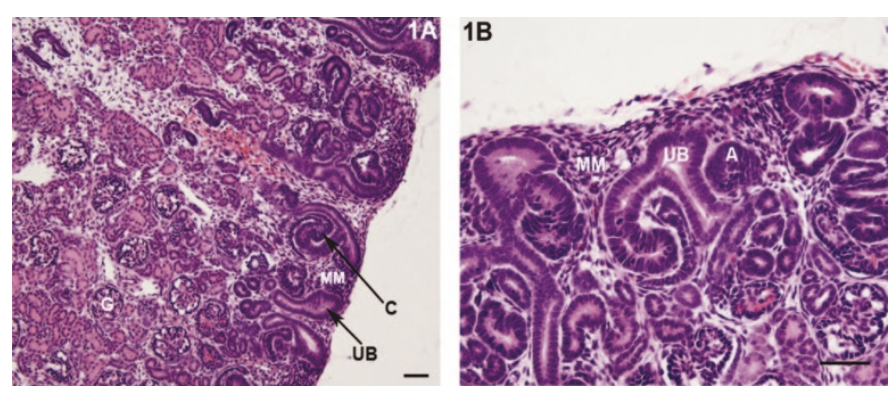

Figure 1. Representative light microscope photographs of kidney sections in the cortex of a 125-d-gestation premature baboon. (A) There are immature glomerular and tubular structures in the nephrogenic zone in the outer renal cortex (C, comma-shaped body; MM, metanephric mesenchyme; UB, ureteric bud; G, mature glomerulus). (B) A higher magnification view of a branching ureteric bud with a globular aggregate of mesenchymal cells near the tip (A, aggregate of mesenchymal cells). Bar $=50 \mu \mathrm{m}$. 

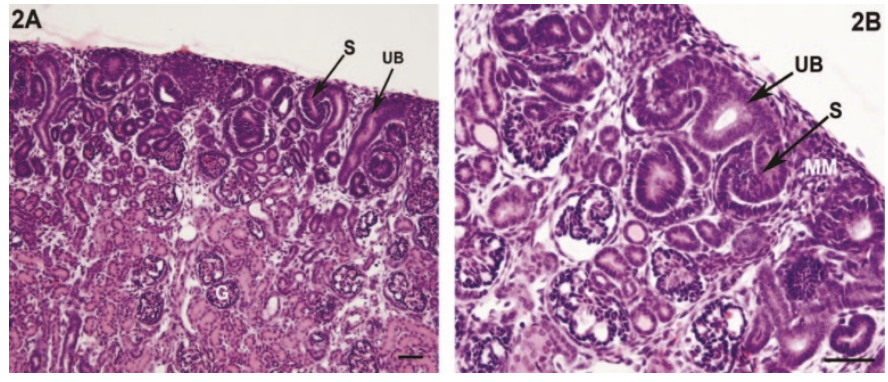

Figure 2. Representative light microscope photographs of kidney sections in the cortex of a 140-d-gestation premature baboon. (A) There are immature glomerular and tubular structures in the nephrogenic zone in the outer renal cortex (S, S-shaped body). (B) A higher magnification view of an S-shaped body and the ureteric bud. Bar $=50 \mu \mathrm{m}$.

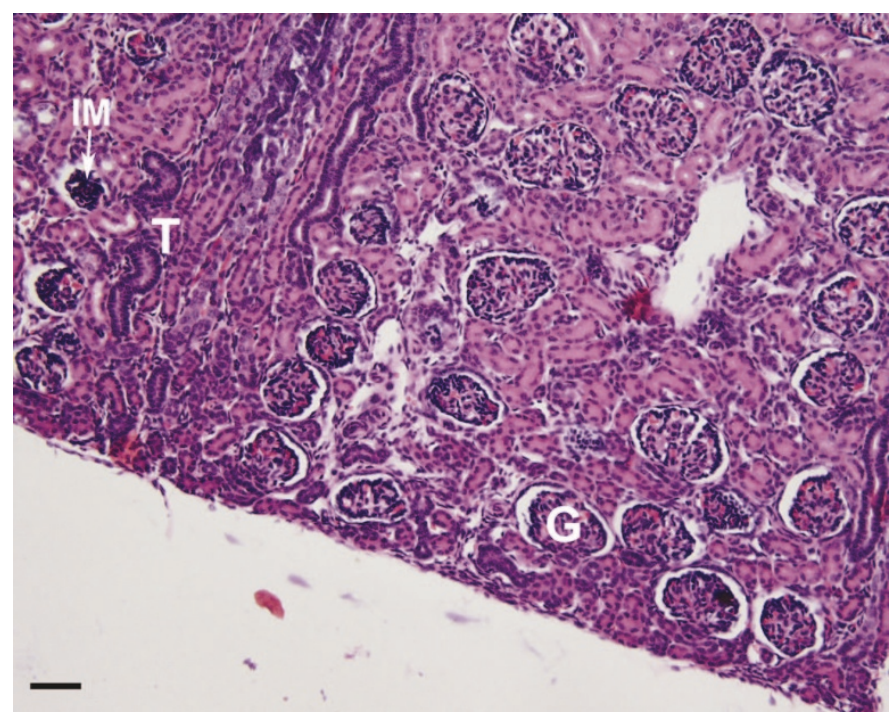

Figure 3. A representative light microscope photograph of a kidney section in the cortex of a 175-d-gestation premature baboon. The glomeruli in the outer renal cortex all are mature except for a few small immature glomeruli $(\mathrm{T}$, maturing tubules; IM, immature glomerulus). Bar $=50 \mu \mathrm{m}$.

Table 1 shows individual birth weights, fixed kidney weights, nephron numbers, kidney volumes, and glomerular and renal corpuscle volumes in baboons that were delivered at 125, 140, 175 , and 185 d gestation.

Linear regression analysis showed a significant correlation between birth weight and kidney weight $\left(R^{2}=0.578, p=\right.$ $0.03)$ and between birth weight and kidney volume $\left(R^{2}=\right.$ $0.540, p=0.04)$. A direct significant relationship was also observed between gestational age and nephron number $\left(R^{2}=\right.$ $0.589, p=0.03$ ). However, because nephrogenesis was observed to be complete by $175 \mathrm{~d}$ gestation, nephron number is not expected to increase after this time point. Nephron number also increased with increasing birth weight $\left(R^{2}=0.562, p=\right.$ $0.03)$.

A highly significant correlation was found between kidney size and the number of nephrons (Fig. 5). Kidney weight was very significantly related to nephron number $\left(R^{2}=0.918, p=0.0002\right.$; Fig. $5 A$ ). In addition, kidney volume was strongly associated with nephron number $\left(R^{2}=0.837, P=0.001\right.$; Fig. $\left.5 B\right)$.

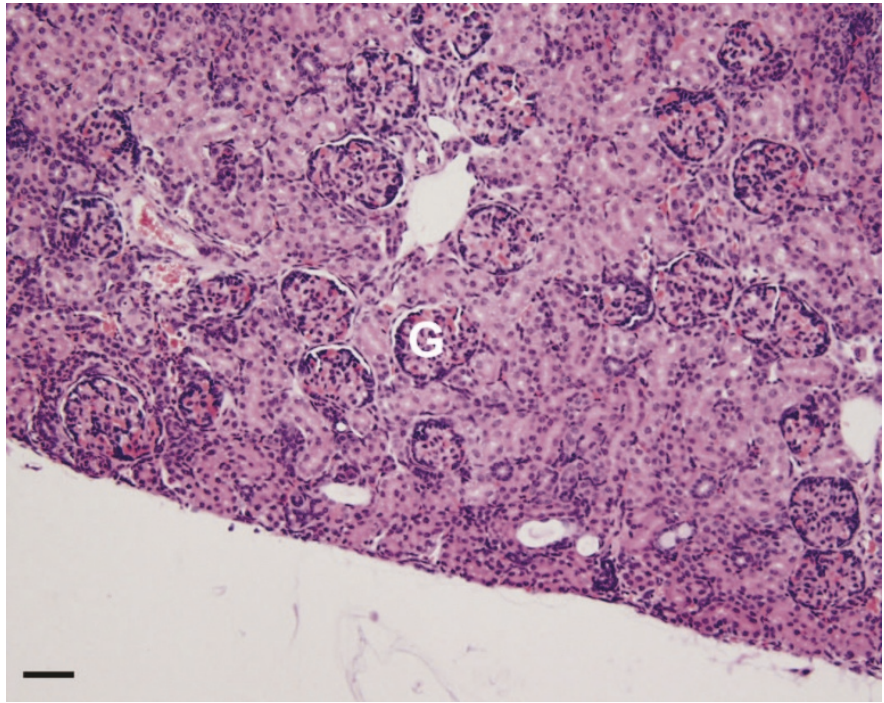

Figure 4. A representative light microscope photograph of a kidney section in the cortex of a 185-d-gestation (term) baboon. The glomeruli in the outer renal cortex all are mature. Bar $=50 \mu \mathrm{m}$.

\section{DISCUSSION}

The present study demonstrated that nephrogenesis in the baboon is complete before term, by 175 d gestation. However, in baboons that were delivered prematurely at 125 and $140 \mathrm{~d}$ gestation, nephrogenesis was still ongoing. Thus, our findings demonstrate that the timing of nephrogenesis in the baboon is similar to humans; thus, it is a good model to study human kidney development. Another important finding of this study is the highly significant correlation between kidney size and nephron number, suggesting that the size of the kidney during normal pregnancy is an important determinant of nephron number.

The present study showed that nephrogenesis in the baboon, like in humans, is complete before term. In humans, $\sim 60 \%$ of nephron formation occurs during the third trimester (12), and it has also been shown that maximum kidney growth occurs at 26-34 wk (27). It seems that also in baboons, the majority of nephrons are formed during the last trimester, as indicated from the results of the current study. With full length of gestation in the baboon being $\sim 185 \mathrm{~d}, 125 \mathrm{~d}$ gestation is the beginning of the third trimester. As demonstrated in this study, the number of nephrons in the 125-d-gestation baboon was $\sim 25 \%$ of the number of nephrons in the term baboon, suggesting that the majority of the nephrons are formed after $125 \mathrm{~d}$. Consequently, premature delivery is expected to have detrimental effects on nephrogenesis.

A major finding of the current study was the highly significant relationship between kidney size and nephron number. Both kidney weight and kidney volume demonstrated strong correlations with the number of nephrons $\left(R^{2}=0.918, p=\right.$ $\left.0.0002 ; R^{2}=0.837, p=0.001\right)$. The regression coefficient predicted an increase of 159,671 nephrons per gram of increase in kidney weight. Hence, it seems that renal size is a strong determinant of nephron number in normal pregnancy, and fewer nephrons are expected in a smaller kidney. Indeed, the large increase in nephron number in one of the baboons at 
Table 1. Birth weights, fixed kidney weights and stereological estimates of kidney volumes, nephron numbers, glomerular volumes and renal corpuscle volumes in baboons delivered at 125, 140, 175 and 185 days gestation

\begin{tabular}{|c|c|c|c|c|c|c|}
\hline $\begin{array}{c}\text { Days } \\
\text { Gestation }\end{array}$ & $\begin{array}{c}\text { Birth } \\
\text { weight }(\mathrm{g})\end{array}$ & $\begin{array}{c}\text { Kidney } \\
\text { weight }(\mathrm{g})\end{array}$ & $\begin{array}{l}\text { Nephron } \\
\text { number }\end{array}$ & $\begin{array}{c}\text { Kidney } \\
\text { volume }\left(\mathrm{mm}^{3}\right)\end{array}$ & $\begin{array}{c}\text { Mean Glomerular } \\
\text { Tuft volume } \times \\
10^{-4}\left(\mathrm{~mm}^{3}\right)\end{array}$ & $\begin{array}{c}\text { Mean Renal } \\
\text { Corpuscle volume } \\
\times 10^{-4}\left(\mathrm{~mm}^{3}\right)\end{array}$ \\
\hline 125 & 299 & 1.071 & 108,619 & 550.52 & 3.604 & 3.614 \\
\hline 125 & 448 & 1.213 & 122,433 & 646.65 & 4.288 & 4.421 \\
\hline 140 & 540 & 1.651 & 164,525 & 846.52 & 3.525 & 3.722 \\
\hline 175 & 1,163 & 2.594 & 334,316 & $1,637.29$ & 3.361 & 3.627 \\
\hline 175 & 973 & 1.658 & 193,983 & 947.65 & 2.987 & 3.465 \\
\hline 175 & 1,148 & 1.932 & 255,537 & $1,027.56$ & 2.780 & 2.986 \\
\hline 185 & 974 & 2.981 & 427,471 & $1,855.26$ & 3.058 & 3.345 \\
\hline 185 & 910 & 2.241 & 212,716 & $1,503.67$ & 6.363 & 6.515 \\
\hline
\end{tabular}
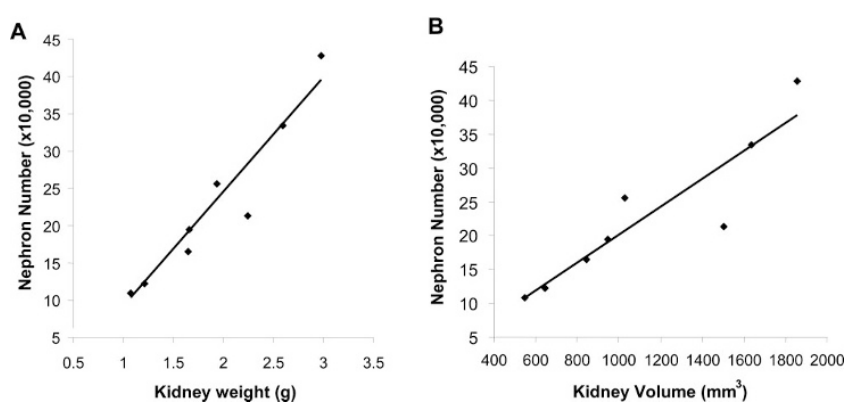

Figure 5. (A) Linear regression analysis of kidney weight $v s$ nephron number in fetal baboons that were delivered at $125,140,175$, and $185 \mathrm{~d}$ gestation. $\left(\mathrm{R}^{2}\right.$ $=0.918, \mathrm{P}=0.0002)(B)$ Linear regression analysis of kidney volume $v s$ nephron number in fetal baboons that were delivered at 125, 140, 175, and 185 d gestation $\left(\mathrm{R}^{2}=0.837, \mathrm{P}=0.001\right)$.

$185 \mathrm{~d}$ gestation, when compared with the other baboon at $185 \mathrm{~d}$ gestation and the baboons at $175 \mathrm{~d}$ gestation can be accounted for in part by the large size of the kidneys in this animal. Although there was only a $6 \%$ difference in body size between the two baboons at $185 \mathrm{~d}$ gestation, there was a $25 \%$ difference in kidney size. Hence, the large difference in nephron number between the two kidneys from $185 \mathrm{~d}$ gestation seems to be due mainly to the large variation in kidney size. In addition, the findings were confirmed with a variability of $7 \%$ between two nephron counts.

It is unknown whether preterm birth leads to a significantly low nephron endowment. In the present study, there was a direct relationship between gestational age and nephron number $\left(R^{2}=0.589, p=0.03\right)$, with the regression coefficient predicting a gain of 3195 nephrons per day of gestations after 125 d. However, because nephrogenesis was complete by $175 \mathrm{~d}$, it is expected that nephron number would increase after $175 \mathrm{~d}$. As nephrogenesis predominantly occurs in late gestation, it is essential to determine whether nephrogenesis continues after preterm delivery and whether nephron endowment is significantly reduced in preterm individuals. Such studies in humans can be performed only in deceased infants, with interpretations of data from such individuals confounded by the postnatal complications leading to their subsequent death. One such study has been performed in preterm infants in which nephrogenesis was shown to continue for a short period after premature birth (28). Renal autopsy tissue was studied from 56 extremely premature infants (birth weight $<1000 \mathrm{~g}$ ), with gestational age ranging from 24 to $27 \mathrm{wk}$ and 10 full-term infants with normal birth weight. The premature infants were divided into two groups, the short survival group that lived for $<40 \mathrm{~d}$ and the long survival group that lived for $>40 \mathrm{~d}$ after birth. The infants were subdivided further into groups with and without renal failure. Nephrogenesis, as indicated by the presence of S-shaped and comma-shaped immature glomeruli, were present only in all preterm infants who survived $<40 \mathrm{~d}$ with or without renal failure and was absent in the longer surviving infants. However, the extremely premature neonates received nephrotoxic antibiotics that may have impaired nephrogenesis in the longer surviving neonates. Radial glomerular counts, obtained by counting the layers of glomeruli, were significantly decreased in all preterm infants compared with full-term control infants and strongly correlated with gestational age.

\section{CONCLUSION}

In conclusion, the results from the present study demonstrate that the baboon is an ideal animal model for studies of human kidney development and, in particular, to study the effects of preterm delivery on nephrogenesis.

Acknowledgments. We thank the Southwest Foundation for Biomedical Research (San Antonio, TX) for provision of the baboon kidneys and Jacqueline Coalson and Vicki Winter from the University of Texas Health Science Center for assistance in obtaining the kidneys and advice in preparation of the manuscript.

\section{REFERENCES}

1. Lumley J 2003 Defining the problem: the epidemiology of preterm birth. Br J Obstet Gynecol 110:3-7

2. Lemons J, Bauer C, Oh W, Korones SB, Papile LA, Stoll BJ, Verter J, Temprosa M, Wright LL, Ehrenkranz RA, Fanaroff AA, Start A, Carlo W, Tyson JE, Donovan EF, Shankaran S, Stevenson DK; NICHD Neonatal Research Network 2001 Very low birth weight outcomes of the National Institute of Child Health and Human Development Neonatal Research Network, January 1995 through December 1996. Pediatrics 107:E1

3. Bendon RW, Coventry S 2004 Non-iatrogenic pathology of the preterm infant. Semin Neonatol 9:281-287

4. Drukker A, Guignard JP 2002 Renal aspects of the term and preterm infant: a selective update. Curr Opin Pediatr 14:175-82

5. Jahnukainen T, Chen M, Berg U, Celsi G 2001 Antenatal glucocorticoids and renal function after birth. Semin Neonatol 6:351-355

6. Haycock GB 1998 Development of glomerular filtration and tubular sodium reabsorption in the human fetus and newborn. Br J Urol 81:33-38

7. Kuure S, Vuolteenaho R, Vainio S 2000 Kidney morphogenesis: cellular and molecular regulation. Mech Dev 92:31-45

8. Moritz KM, Dodic M, Wintour EM 2003 Kidney development and the fetal programming of adult disease. Bioessays 25:212-220 
9. Moritz KM, Wintour EM 1999 Functional development of the meso- and metanephros. Pediatr Nephrol 13:171-178

10. Almeida JR, Mandarim-de-Lacerda CA 2002 Quantitative study of the commashaped body, s-shaped body and vascularized glomerulus in the second and third human gestational trimesters. Early Hum Dev 69:1-13

11. Clark AT, Bertram JF 1999 Molecular regulation of nephron endowment. Am J Physiol 276:F485-F497

12. Hinchliffe SA, Sargent PH, Howard CV, Chan YF, van Velzen D 1991 Human intrauterine renal growth expressed in absolute number of glomeruli assessed by the disector method and Cavalieri principle. Lab Invest 64:777-784

13. Inder TE, Wells SJ, Mogridge NB, Spencer C, Volpe JJ 2003 Defining the nature of the cerebral abnormalities in the premature infant: a qualitative magnetic resonance imaging study. J Pediatr 143:171-179

14. Seidner SR, Jobe AH, Coalson JJ, Ikegami M 1998 Abnormal surfactant metabolism and function in preterm ventilated baboons. Am J Respir Crit Care Med 158:1982-1989

15. Willet KE, Jobe AH, Ikegami M, Kovar J, Sly PD 2001 Lung morphometry after repetitive antenatal glucocorticoid treatment in preterm sheep. Am J Respir Crit Care Med 163:1437-1443

16. Thomson MA, Yoder BA, Winter VT, Martin H, Catland D, Siler-Khodr TM, Coalson JJ 2004 Treatment of immature baboons for 28 days with early nasa continuous positive airway pressure. Am J Respir Crit Care Med 169:1054-1062

17. Koenen SV, Mecenas CA, Smith GS, Jenkins S, Nathanielsz PW 2002 Effects of maternal betamethasone administration on fetal and maternal blood pressure and heart rate in the baboon at 0.7 of gestation. Am J Obstet Gynecol 186:812-817

18. Yoder B, Martin H, McCurnin DC, Coalson JJ 2002 Impaired urinary cortiso excretion and early cardiopulmonary dysfunction in immature baboons. Pediatr Res $51: 426-432$
19. Ervin MG, Seidner SR, Leland MM, Ikegami M, Jobe AH 1998 Direct feta glucocorticoid treatment alters postnatal adaptation in premature newborn baboons. Am J Physiol 274:R1169-R1176

20. Ervin MG, Berry LM, Ikegami M, Jobe AH, Padbury JF, Polk DH 1996 Single dose fetal betamethasone administration stabilizes postnatal glomerular filtration rate and alters endocrine function in premature lambs. Pediatr Res 40:645-651

21. Coalson JJ, Winter V, de Lemos RA 1995 Decreased alveolarization in baboon survivors with bronchopulmonary dysplasia. Am J Respir Crit Care Med 152:640 646

22. Rodriguez CG, Makori N, Cukierski MA, Hendrickx AG 1996 Development of the definitive kidney in the cynomolgus monkey (Macaca fascicularis). J Med Primatol 25:122-132

23. Zoetis T, Hurtt ME 2003 Species comparison of anatomical and functional renal development. Birth Defects Res Part B Dev Reprod Toxicol 68:111-120

24. Gundersen HJ, Jensen EB 1987 The efficiency of systematic sampling in stereology and its prediction. J Microsc 147:229-263

25. Bertram JF 2001 Counting in the kidney. Kidney Int 59:792-796

26. Black M, Briscoe TA, Dunstan HJ, Bertram JF, Johnston CL 2001 Effect of angiotensin-converting enzyme inhibition on renal filtration surface area in hypertensive rats. Kidney Int 60:1837-1843

27. Konje JC, Bell SC, Morton JJ, de Chazal R, Taylor DJ 1996 Human fetal kidney morphometry during gestation and the relationship between weight, kidney morphometry and plasma active renin concentration at birth. Clin Sci (Lond) 91:169-75

28. Rodriguez MM, Gomez AH, Abitbol CL, Chandar JJ, Duara S, Zilleruelo GE 2004 Histomorphometric analysis of postnatal glomerulogenesis in extremely preterm infants. Pediatr Dev Pathol 7:17-25 